\title{
GSM Base Station Subsystem Management Application
}

\author{
Vit Novotny, Pavel Svoboda \\ Faculty of Electrical Engineering and Communication, Dpt. of Telecommunication, \\ Brno University of Technology \\ \{novotnyv@afeec.vutbr.cz, xsvobo63@stud.feec.vutbr.cz\}
}

\begin{abstract}
The Faculty of Electrical Engineering and Communication of the Brno University of Technology offers several subjects focused on mobile communication networks. For practical exercises the experimental mobile network has been building at our faculty. As we do not have OMC or NMC in our network and as the low level network management is quite complex we designed the software that is useful tool for configuration and management of the network on the higher level. The paper deals with our experimental network and with software we have developed.
\end{abstract}

Keywords: GSM, BSS, BSS database, BSS administration

\section{Introduction}

Mobile networks and mobile communication have become very common in many parts of our lives. Even if usage of mobile services becomes simpler and simpler, the mobile network and also its management are more and more complicated. This is due to the more complex management procedures of the network resources allocation that is required by the operators to make network resources allocation much more effective so that the network infrastructure costs are lower and the operator can offer lower prices for its services and to attract more subscribers and to earn more money as the result. Of course also reliability of the network infrastructure and quality of the provided services are very important so that the operator puts quite large investments into the operation and maintenance centres (OMCs) and network management centres (NMC).

\section{Experimental mobile network}

Operators require more and more educated engineers in the area of mobile networks. Therefore also at the Faculty of Electrical Engineering and Communication of Brno University of Technology several subjects specialized on mobile networks are taught. To enable students to get practical experience from the area of mobile networks we have been building experimental mobile network. It is complicated and quite expensive but we hope that we will succeed at the end. 
What we maybe will not manage at all are the OMC and NMC elements. So that when we want to configure the access network and core network elements we need to do it at the low level, e.g. over terminal connection with the control processor of given part of mobile network. Such low level configuration is quite complicated operation and the management staff has to be skilled.

Therefore we decided to design the communication software that will simplify the management process of our experimental network.

Structure of the mobile experimental network at the Faculty of Electrical Engineering and Computer Science is shown in Fig. 1. It consists of two main parts base station subsystem BSS and network switching system NSS. It can be seen that it is incomplete. Nevertheless the GSM part, i.e. circuit switched has been already put into operation. The main element of network subsystem, i.e. MSC is not physically equipped in the network, but it is simulated by the Tekelec simulator which also simulates functionality of Home Location Register (HLR), Visitor Location Register (VLR) and Authentication Centre (AuC).

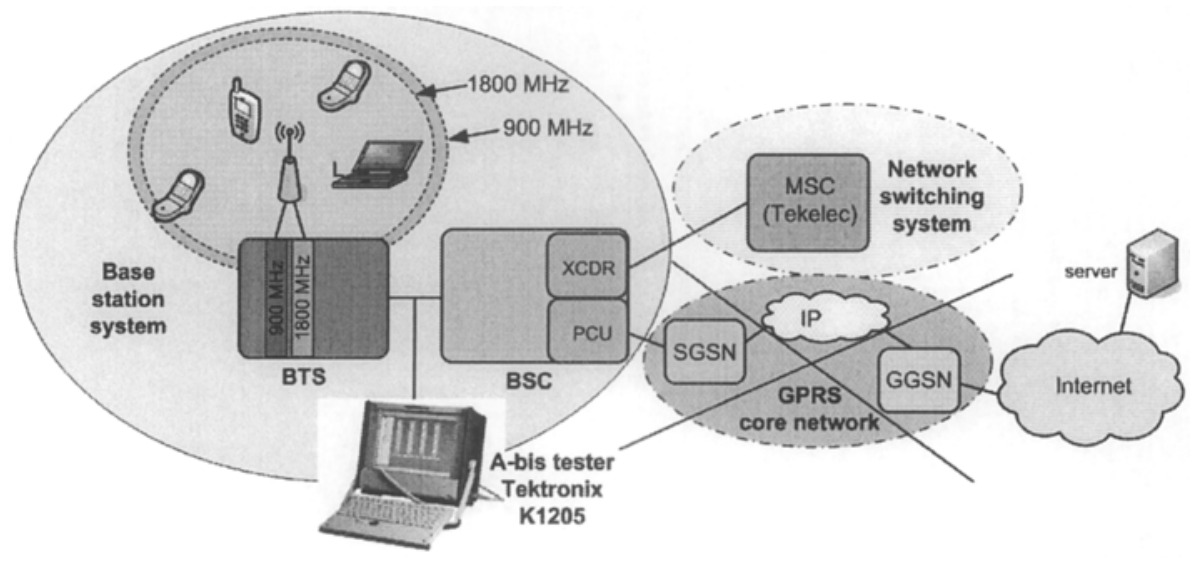

Fig. 1. Experimental mobile network

Building of GPRS network is at the beginning because only Packet Control Unit PCU is equipped. We suppose to equip other GPRS components during next two years and put it into full operation.

Our experimental network is equipped mainly by components manufactured by Motorola company, see Fig. 2. More precisely the BTS, BSC with transcoder and the PCU components are the products of Motorola company ([2]). 
a)

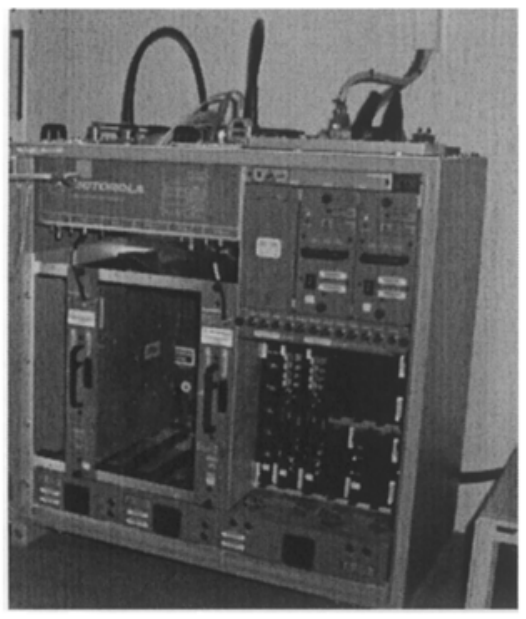

Fig. 2. a) BTS Motorola Horizonmacro ${ }^{\mathrm{TM}}$, functionalities

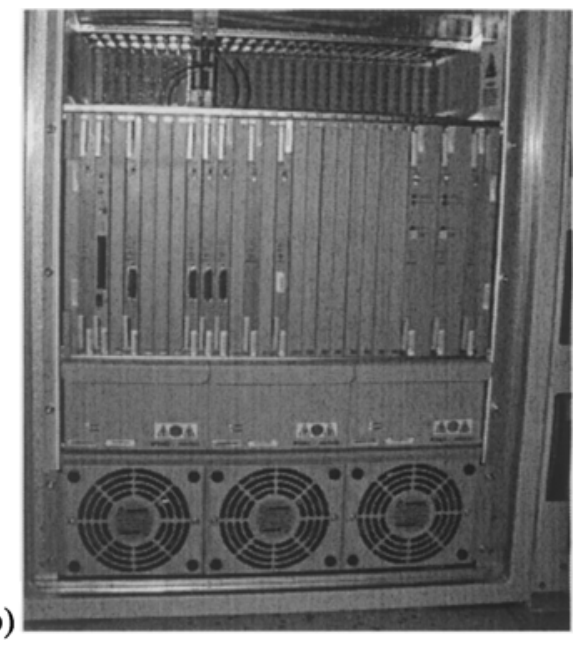

b) $\mathrm{BSSC} 2$ cage equipped by $\mathrm{BSC}$ and $\mathrm{XCDR}$

\section{BSS database}

BSS database is a code object which specifies the hardware equipment of the BSS, hardware functionality and the configuration parameters of the hardware equipment, so that it specifies the overall behaviour of the BSS ([1]). The database can be configured and modified via configuration script. Complete configuration script has to have certain structure so that it contains several configuration steps which are displayed in Fig. 3.

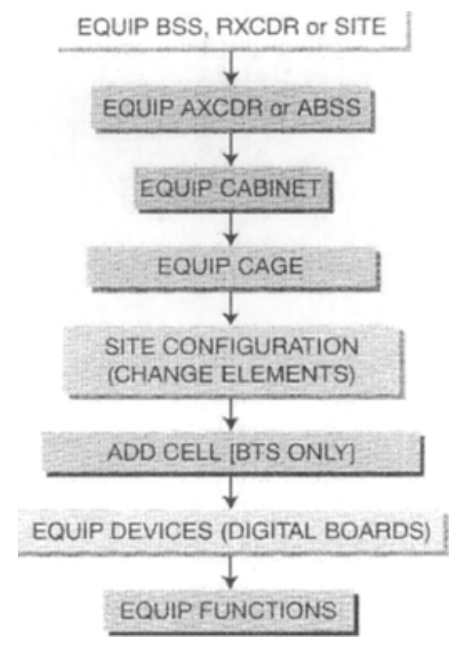

Fig. 3. Database programming sequence 
The database can be changed via MMI (Man-Machine Interface) interface either from the $\mathrm{OMC}$ or from the terminal physically connected to the M-GPROC (master) in the BSC. Database changes are controlled by the CM (Configuration Management) process and by $\mathbf{C A}$ (Central Authority) process that proves or refuses the requested changes. To realize the command appropriate security level is required. There are three security levels protected by passwords. When the command is accepted all changes have to be distributed to all control equipment of the BSS to put them in operation. The commands are necessary to enter in certain order because majority of them depend on the status of equipage of the BSS by previous commands. Therefore the BSS system units should be equipped in order that is shown in Fig. 4.

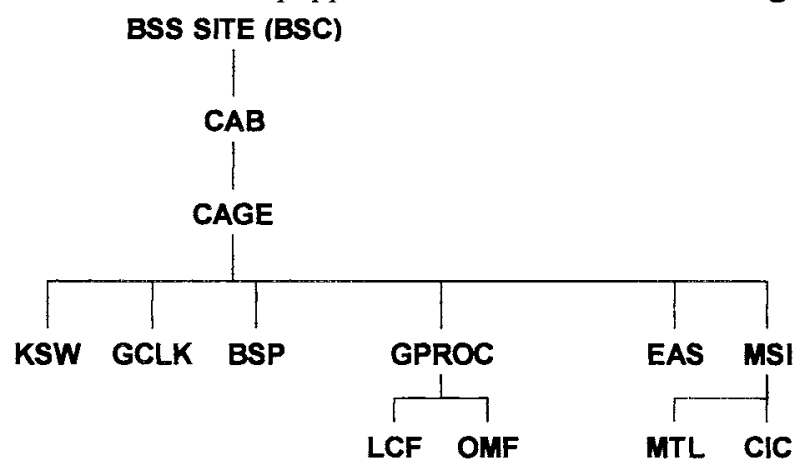

Fig. 4. Hierarchical system for the order of equipping different parts of BSS system by command equip

Two examples of commands follow. First one shows the equipage of the BSC by Kiloport Switch (TDMA switch) and the second one shows the equipage of the BSC by processor card GPROC.

\section{equip bsc KSW}

0

0

0

equip bsc GPROC

1

0

19 it specifies Kiloport Switch module in the BSC cage

0-3-TDM position identifier for KSW

0 or $1-\mathrm{KSW}$ module position, $0=$ slot $27,1=$ slot 1

0-15 - unit number that contains KSW module

GPROC module specification

1-111 - GPROC identifier

0-13 - unit identifier where the GPROC is located

$0-15-$ slot number in the unit 


\section{Management application}

The primary goal of the application is to simplify management of the base subsystem of the GSM mobile network. The application provides BSS administrator help in the form of the syntax and set of valid parameters for required command. Therefore the BSS administrator does not need very expensive OMC centre or to remember syntax of all commands that the administrator otherwise must know to administer the BSS from command line over terminal connection. Therefore the administration is much simpler and faster. The application was written in $\mathrm{C}++$ in integrated development environment Borland ${ }^{3} \mathrm{C}++$ Builder $^{\mathrm{TM}}$ version 6.0 . The application is 32 -bit and it was designed for Windows ${ }^{\circledR}$ operating systems.

\subsection{Libraries and components}

Standard libraries and components of $\mathrm{C}++$ Builder $^{\mathrm{TM}}$ integrated development environment were used. BSS commands, their parameters and help texts are stored in the Paradox 7 format and the access to it is realized over standard database components. Either SQL interface is used to store or to get information or thick client is used direct access.

Application communicates with the base subsystem over serial interface RS232-C. ComPort Library v.3.0 by Dejan Carnilla is used to serve the communication.

\subsection{Program structure}

The application consists of several form and dialog windows and of one data module. The main interface window is shown in Fig. 5. Majority of operations are realized via this window. The other forms windows are used to access to the datbase tables containing commands and their parameters. The other window is displayed when the help contains a lot of text and it is valuable to present it in own window. Remaining windows are used for configuration of the program parameters and to display some supplementary information.

When the program starts the main window is displayed. Data module fills ComboBox components by the appropriate data, i.e. by commands, their parameters and other auxiliary values. At the bottom of the main window the most frequently used parameters are displayed to easily revoke them whe necessary.

The parameters for communication via serial line are already preset (COM2, 9600 bps, 8 bit words, 1 stop bit and no parity). Of course the parameters can be changed when needed.

The commands are edited in the top edit box of the main window. The command can be inserted manually or selected from the ComboBox bellow the edit box. When the latter way is used the brief command description appears in the gray frame.

The communication between the application and the BSS is stored in the buffer and displayed in the bottom part of the main window. If convinient the commands are responses exchanged between the program and BSS can be shown in standalone 
window. When necessary they can be exported in text format to the text file and displayed in any text editor when needed.

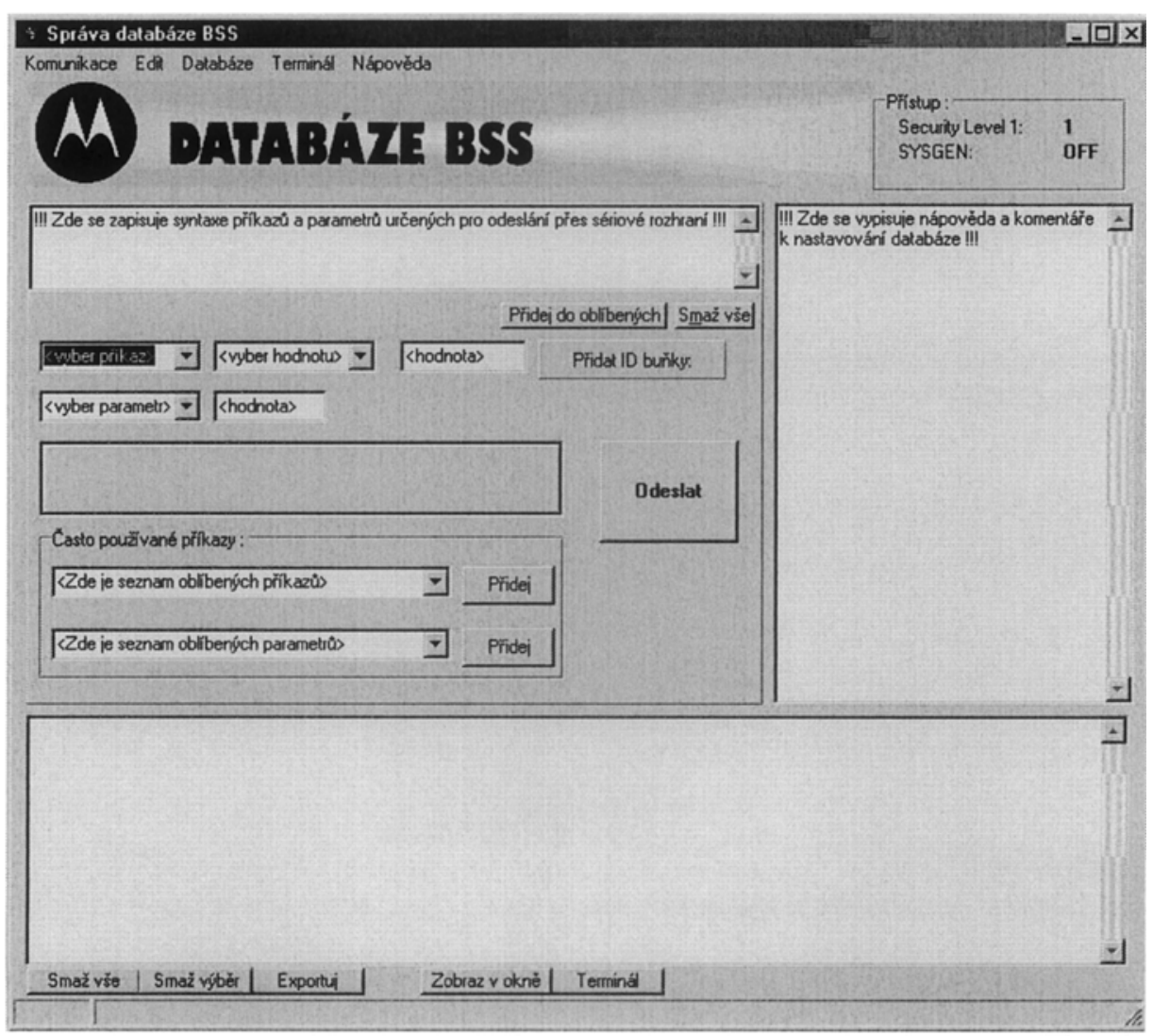

Fig. 5. The main application window

In the case of commands that require the cell ID specification auxiliary form window can used, see Fig. 6.

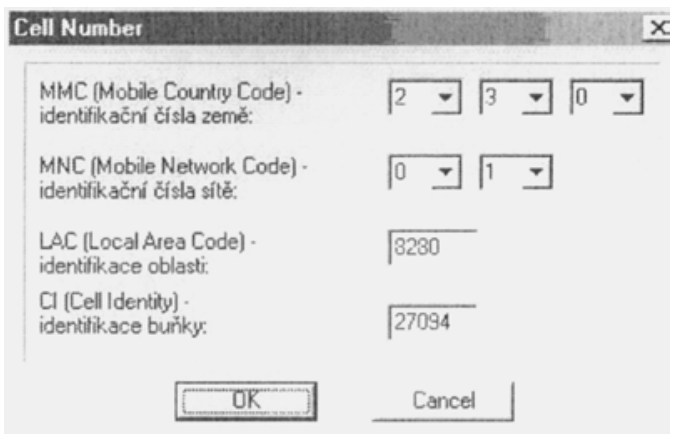

Fig. 6. The form window cell ID specification 
Some commands can be realized only when higher security level is active. There are three security levels and the lowest level is default level. Only small set of commands that change the BSS settings can be run. The user needs to know appropriate password to enter appropriate level. Also some commands can be executed only in Sysgen ON mode. The main window allows the change both of the security level and of the SYSGEN mode, see Fig. 5.

\subsection{Database of command and parameters}

It is possible to manage the database of commands and their parameters. This gives the application flexibility so that it can be quite easily adapted to the other equipment to be configured.

To open the database we select item "Databáze" from the main menu. Then we select the item "Databáze prikkazu a parametrü"and the window shown in Fig. 7. Here the user can modify the database of commands, their arguments and parameters.

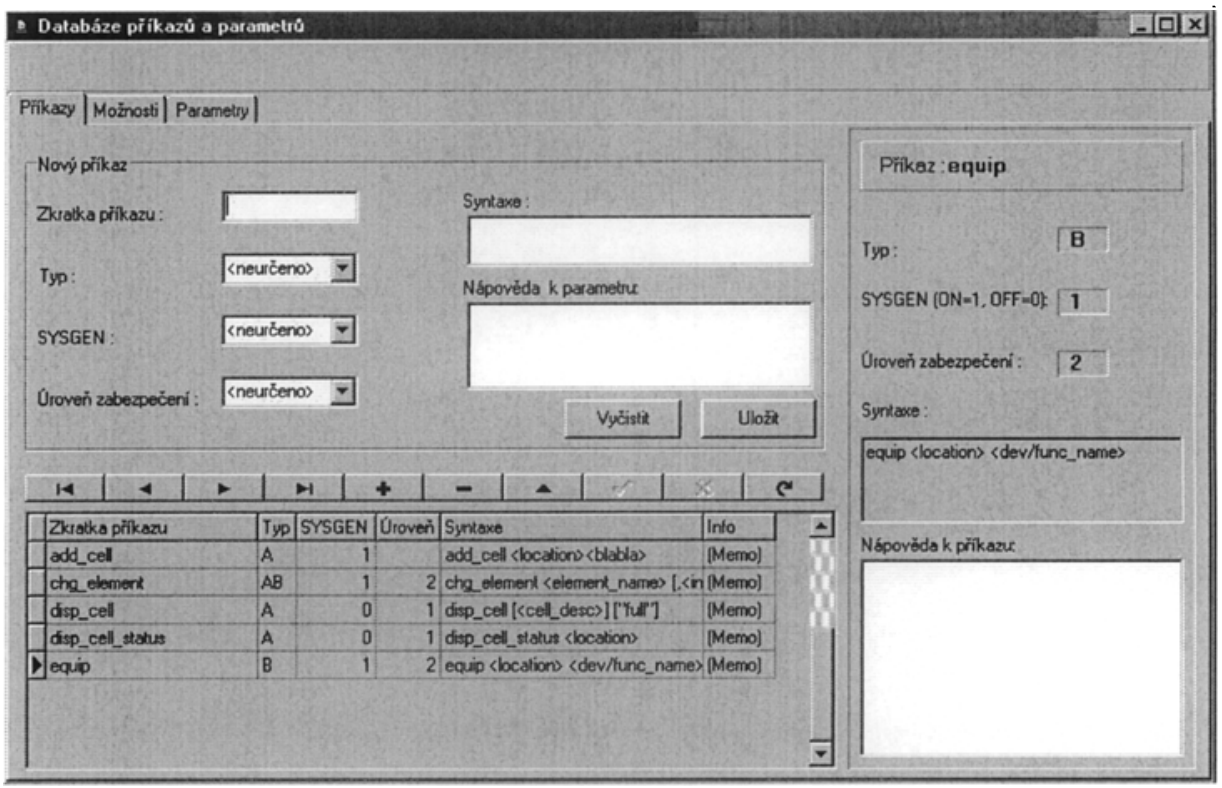

Fig. 7. Window for access to the database

As it can be seen from Fig. 7 also other important information can be added. Mainly the syntax of the command, then help text and also the SYSGEN mode and the security level needed to proceed the command.

To store frequently used commands not only for current session duration but for later use is also very helpful function. This operation is realized via pressing button "Přidej do oblibených" in the main window of the application (Fig. 5). Selected command is analysed and SYSGEN mode and security level is determined. Then the command is stored. New window displayed as shown in Fig. 8. Table is sorted in alphabet order of the command as default. 


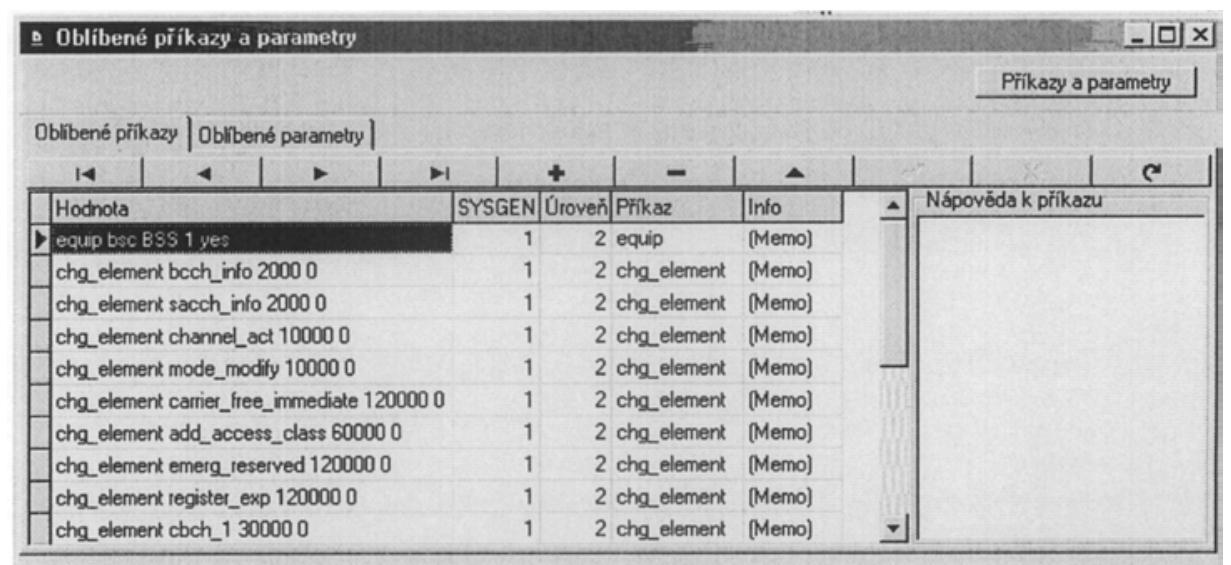

Fig. 8. Window for access to the database of frequently used commands

\subsection{Command exchange}

After the communication parameters are set the program automatically sends "Carriage return" character and the system should display prompt, e.g. "[29/04/07 17:08:26] MMI-RAM $0115 \rightarrow$ " as the response. Then the user can enter the appropriate command or set of commands and when button "Odeslat" is pressed the command is sent to the BSS and providing the syntax is correct, appropriate security level is set and SYSGEN mode is at the required state the command is processed.

\section{Conclusion}

Because of the communication network management is more and more complicated we designed the software to simplify the administration of the BSS of our experimental network. The software works well and it helps us to manage BSS in faster way. The application has been designed in flexible way so that it would be not a big problem to adapt it for administration of other equipment.

Acknowledgement. The paper has been worked out under the support of the project granted by Grant Agency of the Czech Republic No 102/06/1569

\section{References}

1. MOTOROLA Inc. Maintenance of BSS Database GSR7. Motorola, documentation, Swindon, 2003,

2. MOTOROLA Inc. BSS Operational Theory. Motorola, documentation, Swindon, 2002 
3. SOLDANI,D., MAN,L., CUNY,R. QoS and QoE Management in UMTS Cellular Systems. John Wiley and Sons, ISBN 0-470-01639-6, England, 2006,

4. BATES, R., J., BATES, R., J., B. GPRS: General Packet Radio Service (Professional Telecom), ISBN 0071381880, McGraw-Hill Professional, 2001.

5. CASTRO, J.P. All IP in 3G CDMA Networks: The UMTS Infrastructure and Service Platforms for Future Mobile Systems. John Wiley \& Sons, ISBN 0470853220 , USA, 2004.

6. BOSSE,J.G. Signalling in Telecommunication Networks. John Wiley \& Sons, Inc., 1998, ISBN 0-471-57377-9, New York 1998 